\title{
Photospheric flux cancellation and associated flux rope formation and eruption
}

\author{
L. M. Green ${ }^{1}$, B. Kliem ${ }^{1,2,3}$, and A. J. Wallace ${ }^{1}$ \\ ${ }^{1}$ University College London, Mullard Space Science Laboratory, Holmbury St. Mary, Dorking, Surrey, RH5 6NT, UK \\ e-mail: lmg@mssl.ucl.ac.uk \\ 2 University of Potsdam, Institute of Physics and Astronomy, 14476 Potsdam, Germany \\ ${ }^{3}$ Naval Research Laboratory, Space Science Division, Washington, DC 20375, USA
}

Received 3 June 2010 / Accepted 18 October 2010

\begin{abstract}
Aims. We study an evolving bipolar active region that exhibits flux cancellation at the internal polarity inversion line, the formation of a soft X-ray sigmoid along the inversion line and a coronal mass ejection. The aim is to investigate the quantity of flux cancellation that is involved in flux rope formation in the time period leading up to the eruption.

Methods. The active region is studied using its extreme ultraviolet and soft X-ray emissions as it evolves from a sheared arcade to flux rope configuration. The evolution of the photospheric magnetic field is described and used to estimate how much flux is reconnected into the flux rope.

Results. About one third of the active region flux cancels at the internal polarity inversion line in the 2.5 days leading up to the eruption. In this period, the coronal structure evolves from a weakly to a highly sheared arcade and then to a sigmoid that crosses the inversion line in the inverse direction. These properties suggest that a flux rope has formed prior to the eruption. The amount of cancellation implies that up to $60 \%$ of the active region flux could be in the body of the flux rope. We point out that only part of the cancellation contributes to the flux in the rope if the arcade is only weakly sheared, as in the first part of the evolution. This reduces the estimated flux in the rope to $\sim 30 \%$ or less of the active region flux. We suggest that the remaining discrepancy between our estimate and the limiting value of $\sim 10 \%$ of the active region flux, obtained previously by the flux rope insertion method, results from the incomplete coherence of the flux rope, due to nonuniform cancellation along the polarity inversion line. A hot linear feature is observed in the active region which rises as part of the eruption and then likely traces out the field lines close to the axis of the flux rope. The flux cancellation and changing magnetic connections at one end of this feature suggest that the flux rope reaches coherence by reconnection immediately before and early in the impulsive phase of the associated flare. The sigmoid is destroyed in the eruption but reforms quickly, with the amount of cancellation involved being much smaller than in the course of its original formation.
\end{abstract}

Key words. Sun: activity - Sun: coronal mass ejections (CMEs) - magnetic fields - magnetic reconnection - Sun: photosphere Sun: magnetic topology

\section{Introduction}

Photospheric magnetic flux cancellation is observed as the collision and subsequent disappearance of small scale opposite polarity magnetic fragments in magnetograph data (Martin et al. 1985). It is associated with the submergence of small scale loops, which have a small radius of curvature and can be pulled under the photosphere by the magnetic tension force (Harvey et al. 1999). These small loops are likely to be the product of magnetic reconnection which sets in as the opposite polarity fragments collide (Zwaan 1987; van Ballegooijen \& Martens 1989). Recent work has supported the location of the reconnection associated to flux cancellation to be in the photosphere (Yurchyshyn \& Wang 2001; Bellot Rubio \& Beck 2005), but reconnection at higher levels may also be relevant (Wang \& Muglach 2007). Above the small, submerging loop, magnetic field with a concave-up shape is formed. Depending on the plasma beta in the locality and on the strength of the overlying field, the concave-up field lines will be line-tied and form a so-called bald patch (Titov et al. 1993), or they can relax upward to form a coronal loop.

Cancelling magnetic features are observed along photospheric polarity inversion lines (PIL) throughout the quiet sun, at the periphery of active regions and in active regions. Sustained reconnection and flux cancellation along PILs is of particular interest as it is a mechanism by which helical field lines can be formed from a sheared arcade, building up a flux rope (van Ballegooijen \& Martens 1989).

The study of magnetic flux ropes is relevant to many areas of solar physics and since we are currently unable to directly observe the coronal magnetic field, other observational support for the occurrence of flux ropes must be found. Such support is given if the horizontal component of vector magnetic field data is inversely directed at a bald patch section of the PIL. The concaveup configuration could be produced by field lines at the bottom of a flux rope (Athay et al. 1983; Lites 2005; Canou et al. 2009), particularly if the region is bipolar, so that a double arcade can be excluded. Support for a flux rope is also given if continuous $\mathrm{S}$ shaped (sigmoidal) sources of X-ray and EUV emission, which follow the magnetic field lines, exhibit an inverse crossing of the PIL. This, however, is conclusive only if the sigmoid survives an eruption (Gibson \& Fan 2006; Green \& Kliem 2009), since S shaped field lines in a sheared arcade (Antiochos et al. 1994) can only then be excluded.

Numerical simulations of the bodily emergence of a flux rope indicate that the process essentially stops as the magnetic 
axis of the rope reaches the photosphere, producing a sheared arcade in the corona (Fan 2001). In the situation where the flux rope axis is unable to cross the photosphere, flux cancellation may be an important mechanism by which a stable flux rope can form in situ in the solar atmosphere (Amari et al. 2003, 2010; Aulanier et al. 2010). Observations of flux cancellation can therefore allow the investigation of the location and timing of flux rope formation, and can help answer fundamental questions related to the energy accumulation timescale and the flux content of the rope. These are important questions related to the general evolution of the sun's magnetic field and to the onset and driver of eruptive events.

Sigmoidal regions are sites of flux rope formation (Green \& Kliem 2009; Tripathi et al. 2009) and have a high likelihood of producing an eruption (Canfield et al. 1999) and so observations of flux cancellation in these regions can be used to investigate both the flux rope formation and the evolution toward a loss of equilibrium resulting in a coronal mass ejection (CME). The loss of equilibrium can be described as an ideal MHD instability (Hood \& Priest 1981; Kliem \& Török 2006), as a catastrophe (Forbes \& Isenberg 1991), or as a force imbalance between flux rope and overlying arcade field (Mackay \& van Ballegooijen 2006). The overlying arcade field provides a downward tension force, whilst the Lorentz force in the flux rope points upward. The arcade tension force reduces, whilst the flux rope force grows as the sheared arcade field transforms into a flux rope. A force-free equilibrium of the flux rope/arcade configuration is therefore only possible up to a limiting value of the ratio between these fluxes. This value depends on the configuration of photospheric flux and coronal currents. For four decayed active regions, one of which contains a sigmoid, Bobra et al. (2008), Su et al. (2009) and Savcheva \& van Ballegooijen (2009) found that the force balance will be lost if the axial flux in the rope surpasses a threshold lying in the range $\approx(10-14) \%$ of the active region flux. Recent numerical simulations of eruptions driven by flux cancellation have obtained similarly small values for the fraction of cancelled flux at the onset of eruption, lying in the range (6-10)\% (Aulanier et al. 2010; Amari et al. 2010). A related result is that, generally, a coronal flux rope is unstable if the overlying field falls off sufficiently rapidly with height (van Tend \& Kuperus 1978; Török \& Kliem 2005; Kliem \& Török 2006).

Previously, dimming signatures of eruptions have been used to investigate the flux content of the erupting flux rope and make a link with in situ observations of flux ropes at $1 \mathrm{AU}$ (e.g. Webb et al. 2000; Mandrini et al. 2005; Attrill et al. 2006; Jian et al. 2006). However, the erupting stucture is strongly modified by magnetic reconnection that occurs in the current sheet under the rising flux rope and produces the post-eruption flare arcade (e.g., Qiu et al. 2007). We rather use flux cancellation in the active region to study the flux content of the structure before the eruption onset.

In a small number of cases, the eruption from a sigmoidal active region has been observed as the rise of a faint, nearly linear feature in the soft X-ray emission, suggested to be formed near the magnetic axis of a flux rope (Moore et al. 2001; McKenzie $\&$ Canfield 2008). Alternatively, a recent simulation of an eruption from a sigmoidal active region suggests that this feature may be formed in the current layer that develops in the arcade field above an erupting flux rope (Aulanier et al. 2010).

Here we present observations of a sigmoidal active region where the main phases of the evolution of the magnetic field are observed; from emergence to decay. We investigate the formation of a flux rope via reconnection and cancellation along the internal PIL, and the point at which the overlying arcade field is unable to hold down the flux rope leading to a loss of equilibrium and coronal mass ejection. The evolution of a linear feature observed before and during the eruption is also studied.

\section{Photospheric field evolution}

SOHO/MDI measures the line of sight magnetic field in the midphotosphere (Scherrer et al. 1995) and shows that NOAA active region (AR) 10977 emerges in a quiet sun region which has a dominantly negative polarity.

The evolution of the photospheric flux is monitored by fitting a separate contour around each polarity which is adjusted with time to account for the spatial evolution of the active region. The contour is defined by eye and the data are displayed in such as way as to ensure weak flux fragments can be determined. The data are corrected for the area foreshortening that occurs away from central meridian and the radial field component is estimated using the IDL Solar Software routine zradialise. The latter part of the emergence phase is observed and lasts until around 4 December 06:00 UT. During this time the polarities are imbalanced with more positive than negative flux detected. This is due to a projection effect produced by the presence of a significant horizontal field component as the flux crosses the photosphere which in the eastern (western) hemisphere results in the following (leading) polarity of the active region dominating the flux budget (Green et al. 2003). During the subsequent decay phase the photospheric field evolution is dominated by fragmentation, motion due to supergranular flows and cancellation of flux where opposite polarity fragments collide. This flux cancellation involves field at the internal PIL of the bipolar region as well as surrounding quiet sun field. The dominance of negative polarity in the surrounding field results in a stronger reduction of the region's positive flux than the negative as the cancellation proceeds.

As the active region disperses the flux determined by using a contour increasingly includes a contribution from small-scale fields that presumably connect within the boundary defined for each polarity, rather than across the PIL. This small-scale flux is quantified by measuring the magntitude of positive (negative) flux within the negative (positive) polarity contour. Both polarities of the small-scale flux are then removed from the budget for each contour. The small-scale flux contributes at most $5 \%$ of the active region flux. Approximately $50 \%$ of the active region flux cancels in the time period from the peak flux value on 4 December to the time of the eruption on 7 December (Fig. 1). The dispersal of the region and cancellation of its flux continue through the subsequent days.

In order to investigate how much flux is involved in the building of the flux rope, we consider now only the flux cancellation at the internal PIL. The negative polarity is fragmented throughout the active region's lifetime; however, one main fragment is observed to be involved with ongoing cancellation at the internal PIL in the time leading up to the eruption on 7 December (a fragment to the south cancels thereafter). Figure 2, panels 2-5, shows this fragment within the yellow contour. The cancellation occcurs predominantly at the PIL under the sigmoid centre and can be tracked reliably since the cancellation occurs with positive polarity fragments of the active region and not with the surrounding, and dominantly negative, quiet sun field. The evolution of the active region's positive flux cannot be followed in this way as it is not possible to separate the cancellation with surrounding flux from that at the internal PIL. During the time period from 4 December 19:12 to 7 December 03:12 UT, $0.71 \times 10^{21} \mathrm{Mx}$ of flux is cancelled at the internal PIL (Table 1). 


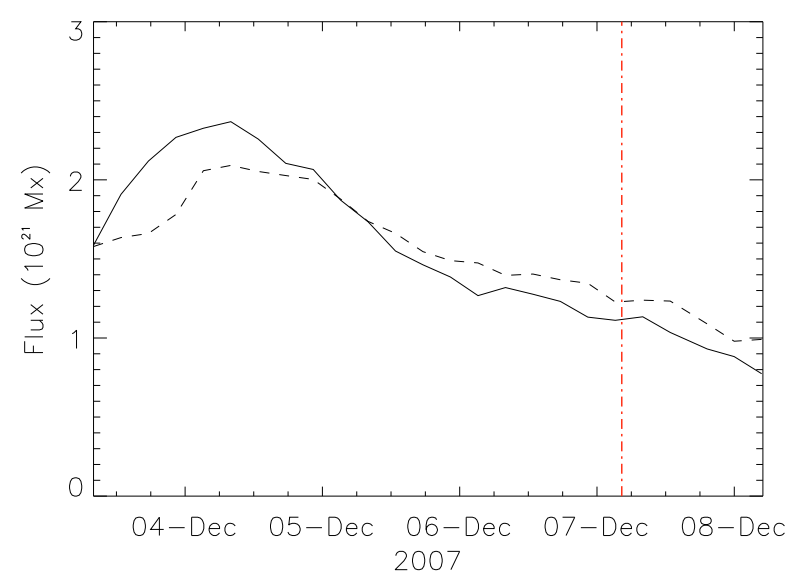

Fig. 1. Flux evolution in AR 10977 as determined by the SOHO/MDI data. The positive (negative) flux is shown by the continuous (dashed) line and the time of the eruption by the red dash-dot line.

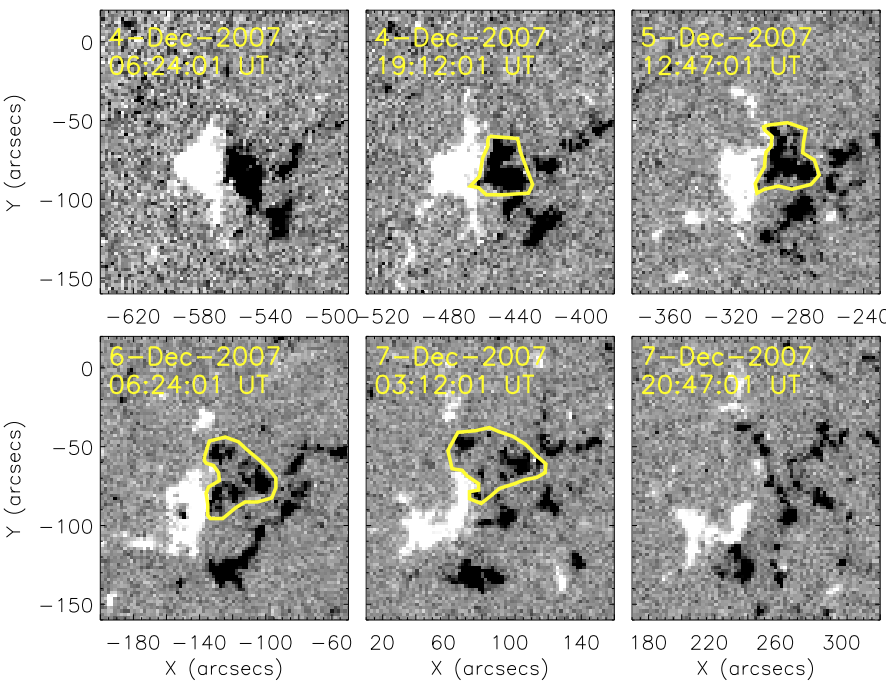

Fig. 2. Photospheric flux evolution in AR 10977 as determined with $S O H O / M D I$ magnetograms. The data are displayed between \pm 100 Gauss. The yellow contour shows the area within which the negative flux is measured during the main flux cancellation episode. In a similar way, the total unsigned flux of the region is determined by fitting a countour around the whole active region.

This represents $\approx 34 \%$ of the peak negative flux value of the active region. Since the cancellation is enabled by reconnection, with flux remaining in the active region (Sect. 6), an amount of flux equal to that cancelled is potentially available for transformation into the flux rope.

At the time of the eruption, the unsigned average flux in the active region was $1.17 \times 10^{21} \mathrm{Mx}$ (see Fig. 1). This flux value includes that in the flux rope body, which intersects the photosphere at the flux rope feet, as well as that of the overlying arcade field.

\section{Formation of sigmoid}

The evolution of the coronal magnetic field structure is studied using data from the X-ray Telescope (XRT) onboard Hinode (Golub et al. 2007) taken with the C Poly filter, which image
Table 1. Flux evolution within the negative fragment that undergoes cancellation at the active region's internal polarity inversion line.

\begin{tabular}{lc}
\hline \hline Date & Flux $\left(10^{21} \mathrm{Mx}\right)$ \\
\hline 4 Dec. 19:12 UT & -1.10 \\
5 Dec. 12:47 UT & -0.82 \\
6 Dec. 06:24 UT & -0.58 \\
7 Dec. 03:12 UT & -0.39 \\
\hline
\end{tabular}
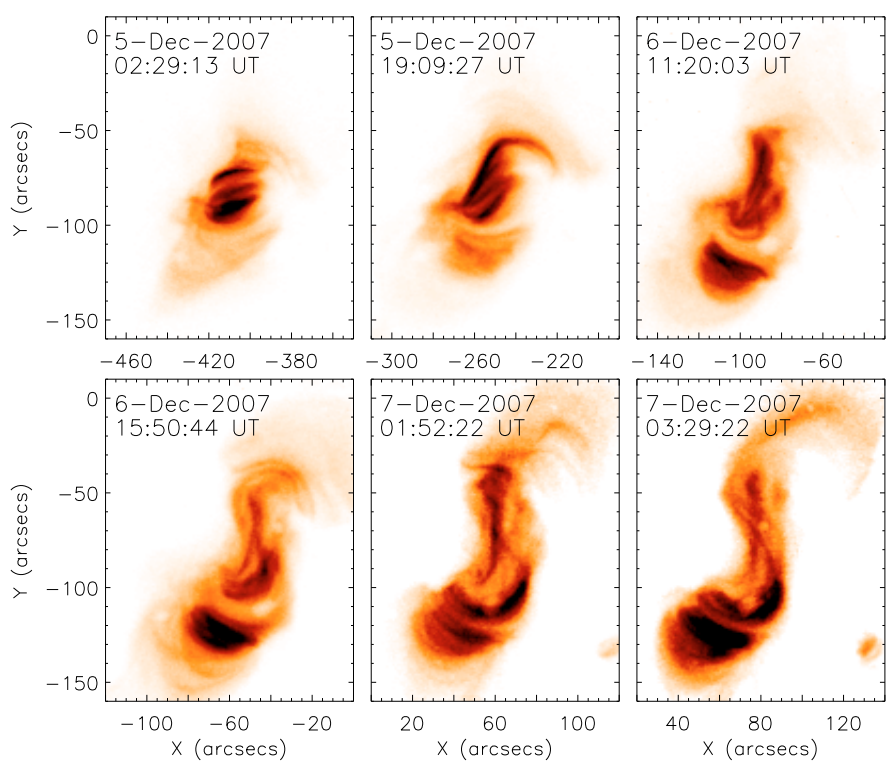

Fig. 3. Hinode/XRT C Poly filter observations of AR 10977 showing the formation of the sigmoid from an increasingly sheared arcade.

plasma between 2 and $10 \mathrm{MK}$. The active region loops had an arcade structure during the emergence phase which became increasingly sheared as the region evolved. During the decay phase the soft X-ray loops became more aligned to the PIL in the northern section of the region, whilst more potential-like arcade field remained in the south (Fig. 3). The major flux cancellation episode took place in the northern region where the shear was increasing.

A diffuse, but already continuous, forward $\mathrm{S}$ sigmoid was observed by 6 December 2007 15:51 UT (Fig. 3, panel 4). The foward $\mathrm{S}$ shape indicates that the region had positive helicity (Pevtsov et al. 1997). 50 min before the eruption, at 03:29 UT on 7 December, the sigmoid extends further to the north and is more defined (Fig. 3, panel 6). Figure 4 shows that the central part of the sigmoid, while largely running along the PIL, crosses the PIL in the inverse direction at $y \approx-75^{\prime \prime}$. At this location the PIL bulges out to the west from its overall diagonal orientation parallel to the middle section of the sigmoid. Much of the cancellation that leads to the formation of the sigmoid is occurring in this area.

The inverse PIL crossing indicates that a flux rope has formed, but is not conclusive evidence, since $S$ shaped field lines with an inverse PIL crossing in the middle can also form when an arcade is sheared (Antiochos et al. 1994). However, since the sigmoid formed as a consequence of shearing and converging motions of similar strength, which led to strong flux cancellation, the formation of a flux rope structure is highly likely (van Ballegooijen \& Martens 1989). The configuration would be better described as a (tangled) arcade only in the case that all 

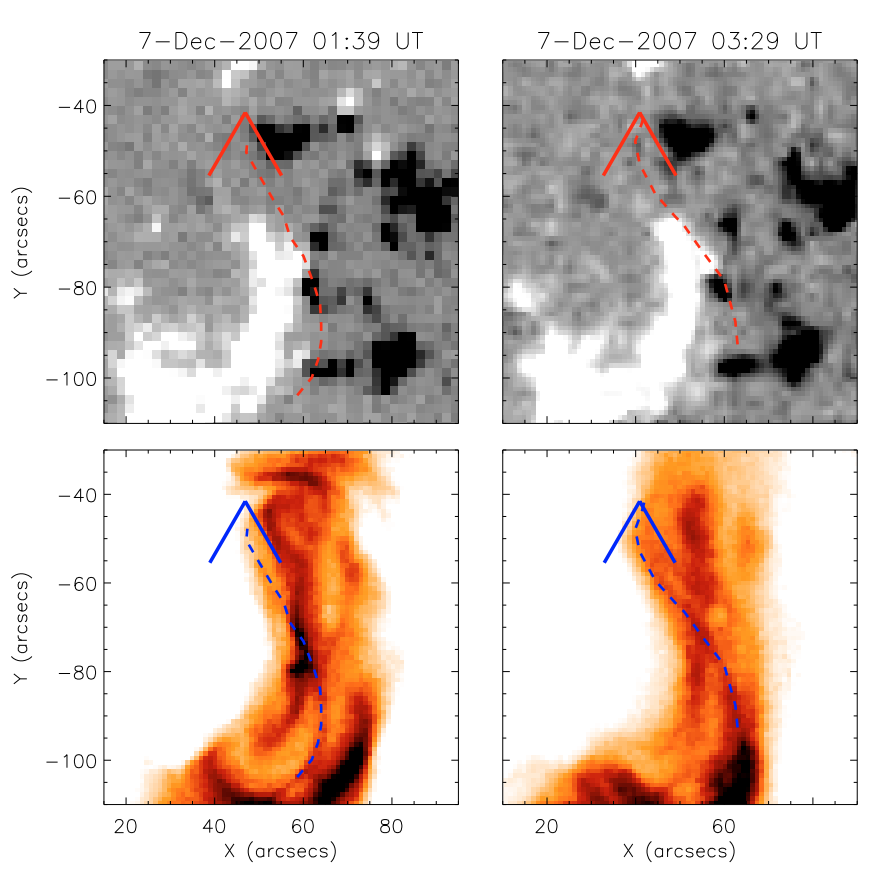

Fig. 4. SOHO/MDI (top row) and Hinode/XRT (bottom row) observations of AR 10977 showing the inversing crossing of sigmoidal threads on 7 December at 01:39 and 03:29 UT. In the second panel the MDI data were taken at 7 December 03:12 UT and rotated to the time of the XRT image at 03:29 UT. The MDI data are displayed between \pm 100 Gauss. The little bipole seen on 7 December after 03 UT at $(x, y)=(135,-130)$ and the corresponding set of compact X-ray loops (Figs. 2 and 3 ) were used to coalign the images. An accuracy of approx. 1.5" is achieved by using this feature as well as other small scale features.

new, initially helical magnetic connections relax upwards into largely concave-down coronal loops. Since this is unlikely, due to the accumulation of axial flux above the PIL by the cancellation (Sect. 6.2.1), we will adopt the flux rope interpretation in the following. Further discussion of the flux rope's magnetic structure is given in Sects. 6.1 and 6.2.2.

The sigmoidal region also has a linear bar-like feature aligned almost north-south and crossing the sigmoid centre and the PIL in the normal direction (panels 4-6 in Fig. 3). The direction of crossing of the PIL indicates that the linear feature is highly sheared arcade-like field (i.e., not bald patch) passing over the forming flux rope in this phase of the evolution.

In the time leading up to the eruption of the sigmoid, there is a westward motion of the southern end of the linear feature which is situated at a latitude of roughly $-90^{\prime \prime}$ to $-100^{\prime \prime}$ and composed of several, only marginally resolved threads. Figure 5 shows that the footpoint motion is occuring during a phase of westward motion and at the beginning of the dispersal of a strong concentration of positive flux in which the field lines are apparently rooted. These motions appear to be driven by the development of a superganular cell (see also panels 4 to 6 in Fig. 2). The positive flux collides with a small negative polarity fragment which cancels during the time period 7 December 00:00 to 06:24 UT. At 7 December 01:39 UT a very small positive fragment has detached from the main concentration at $-95^{\prime \prime}$ (marked by arrows in Fig. 5). It is cancelling with the negative polarity fragment and is largely gone by 04:48 UT. The southern end of a thread in the linear feature follows the path of this positive flux fragment; see the panels at 03:12 UT in Fig. 5, at which time it has become the brightest thread in the feature. The cancellation of the photospheric flux at the southern end of this thread implies that the coronal flux in the thread must join the sigmoid (and then be rooted somewhere along the section of the sigmoid south of the cancellation site). Indeed, by the time the small positive flux fragment has cancelled nearly completely, this thread has formed a new connection with a prominent thread in the sigmoid (see the XRT image at 03:58 UT in Fig. 5 and also the image 20 min later in Fig. 8).

Since the cancelling small positive flux patch is far smaller than the negative flux patch at the northern end of the linear feature, only part of the flux in the linear feature merges with the sigmoid in this phase. The other, probably larger part must remain rooted in the westward moving and dispersing major positive flux patch, which is also clear from the continued presence of threads in the linear feature that end in this area. However, the temporal association with the onset of the CME at about 04:20 UT makes it quite likely that the partial transformation of the arcade-like flux in the linear feature into helical flux in the sigmoid represented the final step toward the destabilization of the configuration. The cancellation of the positive flux patch continues through and beyond the eruption (see Figs. 2 and 5).

The flux added to the sigmoid by the transformed part of the linear feature must run under the magnetic axis of the forming flux rope at the position of the cancellation $\left(y \approx-95^{\prime \prime}\right)$ and above the axis where the linear feature crosses the PIL in the normal direction, which is near the position of the sigmoid's inverse PIL crossing $\left(y \approx-75^{\prime \prime}\right)$. Being wound about the forming flux rope's axis, at least this part of the linear feature must follow the motion of the axis in the subsequent eruption.

The temperature structure of the region is studied using the Extreme ultra-violet Imaging Spectrometer (EIS) onboard Hinode (Culhane et al. 2007). Figure 6 shows simultaneous images in three lines with different formation temperature built from an EIS raster scan immediately before the onset of the CME. The sigmoid is seen at temperatures $\log (T[\mathrm{~K}])=6.1$ to 6.4 , but the linear feature is only seen in the hotter lines imaging plasma at $\log (T[\mathrm{~K}])=6.3$ to 6.4. The EIS data do include flare lines which image at higher temperatures, but the emission is weak at these wavelengths and does not allow the study of the linear feature versus the sigmoid. The high plasma temperature supports the view that part of the linear feature joins the sigmoid through the reconnection that enables the cancellation of the positive flux at its southern end. A sigmoid study by Tripathi et al. (2009) showed that plasma near the axis of the inferred flux rope was cooler than the double-J shaped sigmoid at its edge. This suggests that the linear feature in the present event does not follow a passive bundle of flux near the magnetic axis of the flux rope at this stage of the evolution.

Broadenings of the Fe XV line, which show both the sigmoid and the linear feature clearly, support this view. Figure 7 presents maps of Fe XV radiance and the non-thermal component of the line width (FWHM) from the three available EIS raster scans prior to the eruption which included this line. The non-thermal velocities are calculated using the IDL Solar Software routine eis_width2velocity which removes instrumental width, taken to be $0.057 \AA$ for the $1^{\prime \prime}$ slit Brown et al. (2008), and thermal Doppler width. The broadenings trace out the diagonal part of the $\mathrm{S}$ shaped sigmoid and the linear feature (the whole feature in the first two maps and mainly its southern end in the third map). The implied microturbulence indicates strongly that both structures are sites of ongoing energy release. For the S shaped sigmoid, this supports the now generally held view that such structures form by current dissipation in separatrices (e.g., Titov \& Démoulin 1999). For the linear structure, the 

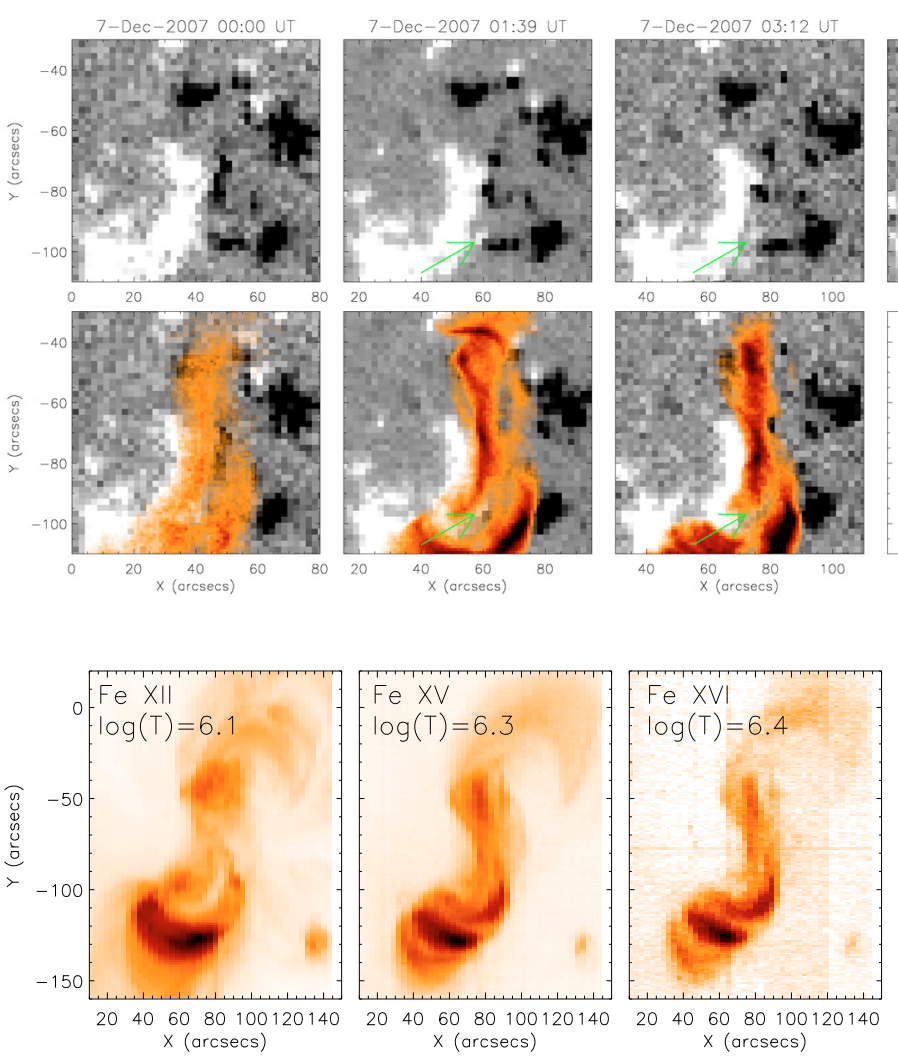

Fig. 6. Hinode/EIS observations of AR 10977 showing the temperature structure of the sigmoid and linear feature. The EIS scan is built from west to east and was taken between 7 December 03:27 UT and 04:19 UT with the $1^{\prime \prime}$ slit in step sizes of $3^{\prime \prime}$.

line width enhancements indicate that this flux interacts with surrounding flux or experiences internal changes. Both are likely, due to the westward motion and due to the dispersal and partial cancellation of the positive flux at the feature's southern end. Accordingly, the line is broadest in this location in the two maps on 7 December, particularly in the map at 03:27 UT. Enhanced widths correlate largely, but not fully, with enhanced radiance of this relatively "hot" line. The third map shows the largest widths in an area of weak emission, and the bright arcade south of the sigmoid (outside the line width maps) has line widths close to the background level within the field of view of the maps. Considering that the EIS scan takes 52 min to build the image of the active region, the location of the cancellation site at $y \approx-95^{\prime \prime}$ will have moved from $x \approx 75^{\prime \prime}$ (as seen in the MDI image at 03:12 UT) to $x \approx 81^{\prime \prime}$ as seen in the EIS image which reaches the location of cancellation at 03:47 UT. The cancellation site shows line width enhancements similar to the main part of the linear feature and the continuous $\mathrm{S}$ shaped sigmoid thread, but it does not stand out as a particular enhancement. This may have several origins: most of this cancellation event proceeds between the two EIS scans; it involves only relatively a small amount of flux; the associated reconnection occurs low in the atmosphere so that the coronal responses remain weak.

\section{Eruption}

The eruption of the flux rope can be seen to occur in two steps; a failed eruption followed $3.5 \mathrm{~h}$ later by a successful eruption which produces the white light CME.

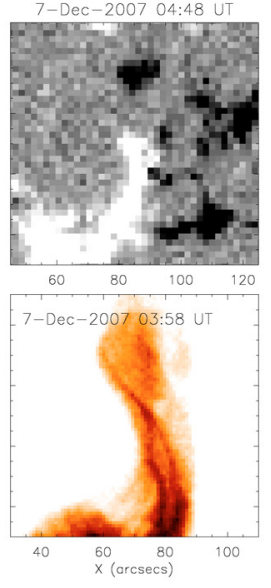

Fig. 5. XRT and MDI overlay (bottom row) showing that part of the linear feature merges with the continuous $\mathrm{S}$ shaped sigmoid as a consequence of the westward motion and partial cancellation of the flux at its southern end (marked by arrows). The fourth panel shows the sigmoid shortly before the eruption. There is no corresponding MDI image available. The same MDI images, including the subsequent one, taken during the eruption, are displayed in the top row for clarity.
The failed eruption occurs while the linear feature begins to transform into the sigmoid and may facilitate the reconnection between the two flux systems. It commences around 7 December 00:45 UT and is seen as the rise of a bundle of field lines at the sigmoid centre (Fig. 8, panel 2 at 00:48 UT). After the failed eruption the active region's overall structure remains the same, but the northern sigmoid elbow (where the top of the $\mathrm{S}$ curves around) has developed and has increased soft X-ray emission (Fig. 9). The XRT images during 03:57-04:19 UT in Fig. 8 show two superimposed sigmoids; one which has the large northern elbow and a second, brighter $\mathrm{S}$ shaped emission trace, which branches off at latitude $\approx-40^{\prime \prime}$.

The successful (ejective) eruption begins in the decay phase of the failed eruption, at approximately 04:20 UT on 7 December 2007 (Fig. 9). It is accompanied by a B1.4-class GOES flare and an EUV wave (Ma et al. 2009). The CME, observed by $\mathrm{SOHO} / \mathrm{LASCO}$, displayed a flux rope structure and propagated westward with a projected velocity of $\approx 300 \mathrm{~km} \mathrm{~s}^{-1}$. The true velocity may have been much higher, since the ejection originated near sun centre.

In the lower corona, the eruption is evidenced by the motion of the linear feature as seen in soft X-rays. This feature, although it is now increasingly curved, can be identified by the isolated negative flux patch at its northern end and moves to the west as does the associated CME (see black arrows in panels 5-7 of Fig. 8). Thus, as argued in Sect. 3, the feature now moves with the rising flux rope's axis. This is essentially in line with the earlier interpretation of bar-like features in Moore et al. (2001) and McKenzie \& Canfield (2008).

Comparing the properties of the linear feature with the current layer in arcade field overlying an erupting flux rope in the simulation by Aulanier et al. (2010), we find differences as well as common aspects. The observed linear feature was present already before the eruption, while the current layer in the simulation formed as a result of the eruption. It rotated in the counterclockwise direction, opposite to the rotation in the simulation for the same chirality of the active region field. Similar to the simulation, the observed linear feature represents a narrow volume of enhanced currents, as indicated by its high temperature and enhanced Fe XV line width. However, we find it to be associated with the overlying arcade-like field only in the phase prior to the eruption, while it must be a part of the flux rope in the course of the eruption. Some of these differences are a consequence of the different formation timescales of the flux rope. In the simulation, the flux rope is fully developed prior to the eruption and the currents within it decrease while it expands in the eruption. 

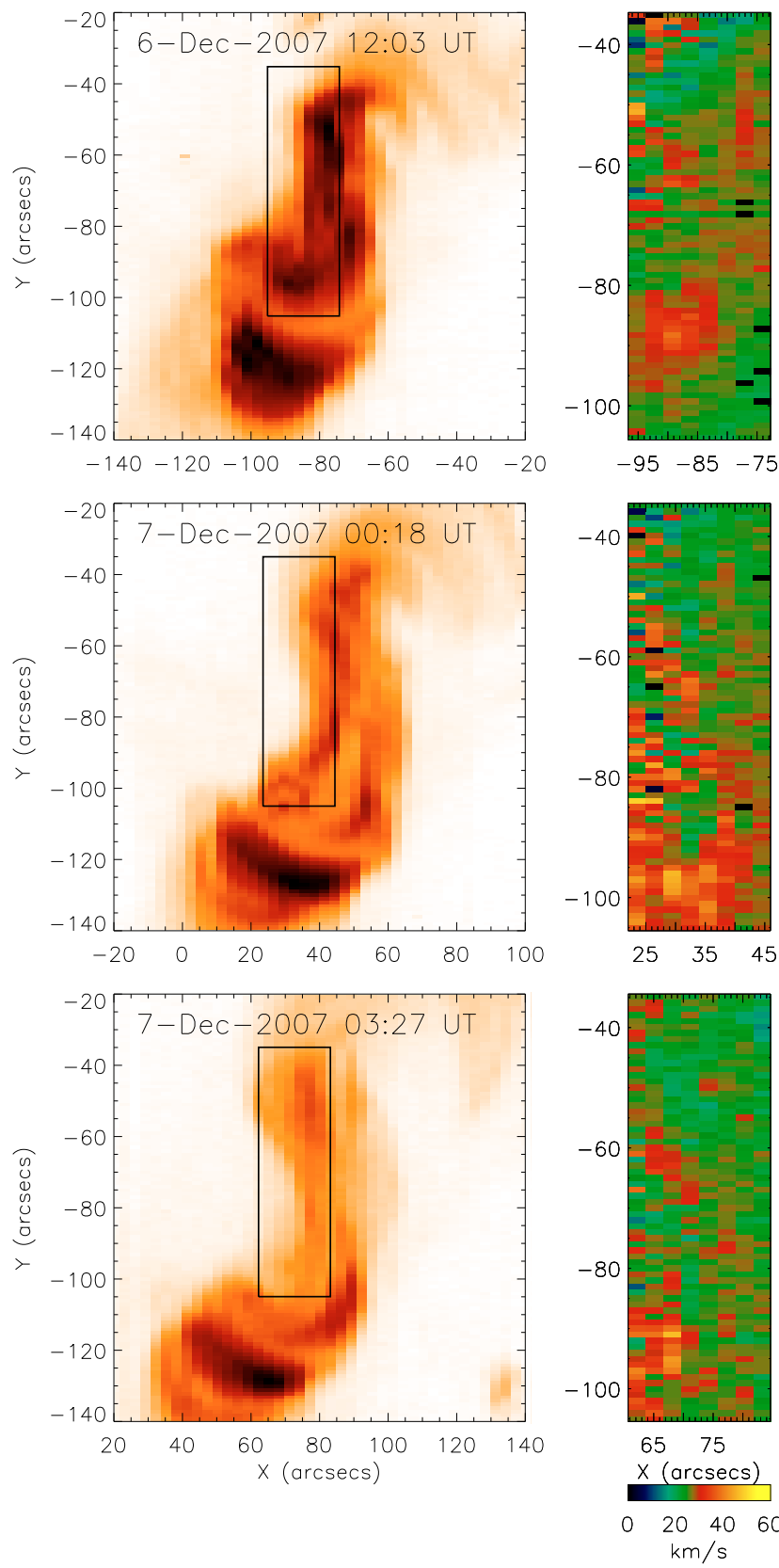

Fig. 7. Hinode/EIS observations of AR 10977 in the Fe XV $284 \AA$ line formed at temperature $2 \mathrm{MK}$. The three EIS scans were taken with the same observation parameters; their start times are given. The left panels display Fe XV radiance and the right panels display the non-thermal component of the line width in the sub-area indicated.

In the event studied here, the overlying flux that threads the linear feature joins the flux of the growing rope only immediately prior to and in the course of the eruption. Thus the linear feature can be related to enhanced currents in overlying field prior to the eruption and to enhanced currents in the flux rope in the course of the eruption.

Under the erupting structure the first, highly sheared posteruption (flare) loops appear. All of these run very closely along the northern section of the sigmoid, passing over the location of the inverse PIL crossing where the major flux rope formation episode has taken place. They branch off from the central S trace of the sigmoid in the range $y \approx-100^{\prime \prime}$ to $-80^{\prime \prime}$. In contrast, the
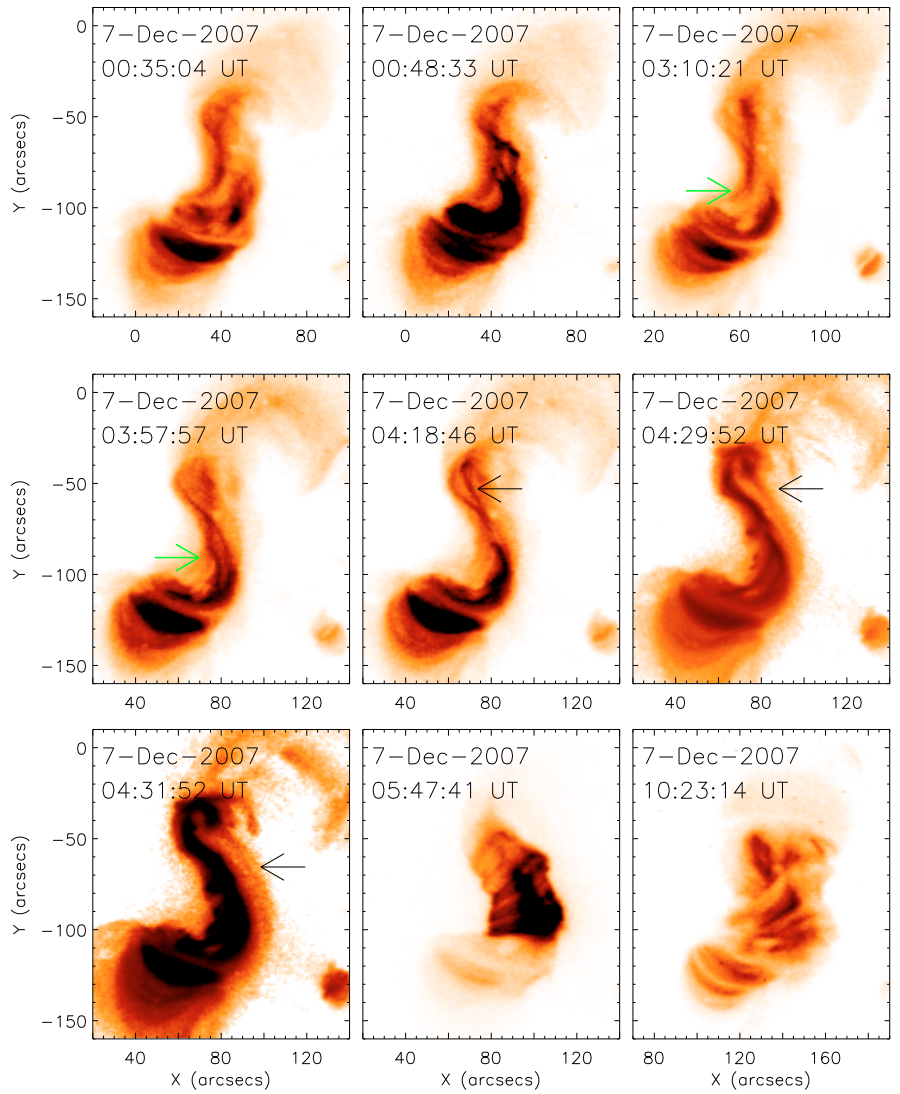

Fig. 8. Hinode/XRT C Poly filter observations showing the failed eruption (top row) and the successful eruption (second and third rows), including the post-eruption configuration (final two panels). Green arrows in panels 3 and 4 indicate the motion of the southern footpoint of the linear feature. Black arrows in panels 5 to 7 indicate the eruption of the linear feature. Panels 6 and 7 have been log displayed to bring out this faint feature.

loops of the flare arcade formed in the gradual phase of the event cross the PIL at nearly right angles (see panels 6 to 8 in Fig. 8).

These data indicate clearly that flare reconnection completed the transformation of the flux in the linear structure into the erupting flux (which is to be expected because the linear feature passed over the centre of the erupting structure immediately before the event). As a result of such reconnection, flare loops must end in the positive flux concentration where the linear feature was rooted before the eruption. Exactly this is the case, starting with the first flare loops at 04:30-32 UT early in the impulsive phase (Fig. 8). Explosive chromospheric evaporation, a further sign of reconnection, commences in this area at $(x, y) \approx\left(70^{\prime \prime},-85^{\prime \prime}\right)$ between 04:30 and 04:35 UT, i.e., also early in the impulsive phase (Chen \& Ding 2010).

A complementary view is obtained by asking where the flux in the erupting rope was rooted. The flare brightenings are largely associated with the smaller and brighter of the two sigmoids, whose northern elbow encloses the strong patch of negative flux at $y \approx-40^{\prime \prime}$ to $-50^{\prime \prime}$, where the linear feature is rooted at its northern end. Figures 2 and 5 show that this is a largely isolated flux concentration. The flux of the erupting rope must largely be rooted in this patch. Hence, the erupting, and now curved, linear feature has become part of the erupting flux rope.

It appears that a trace of the sigmoid remains visible even under the arcade. However, since the region is near Sun centre, this 


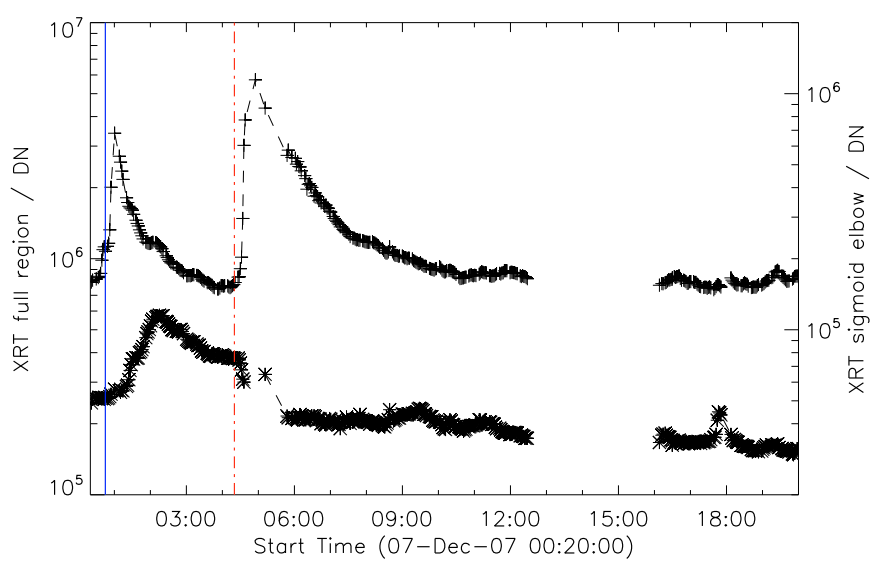

Fig. 9. Light curves of the soft X-ray emission from the whole active region (crosses, top plot) and the northern elbow only (asterisks, bottom plot). The failed eruption beginning around 7 December 00:45 UT (blue solid line) produces a temporary enhancement in active region emission and is followed by an enhancement in the northern arm of the sigmoid. The main eruption (red dash-dot line) is accompanied by enhanced emission in the centre of the active region beginning at 7 December 04:20 UT and reduced emission in the northern elbow.

feature could also result from enhanced brightness at the top of the flare loops, which occurs in some events. The X-ray images immediately after the eruption do not show any clear indications of a surviving sigmoid. Rather, the sigmoid was replaced by two sets of loops, the usual nearly potential arcade in the southern half and a set of highly sheared loops in the northern half (Figs. 8 and 10). The presence of substantial shear immediately after the eruption could indicate that part of the axial flux in the rope survived in the northern section, but it could also result from the high shear of the reclosing field which is indicated by the shape of the first flare loops. The data do not appear to provide a clue which of the two options applies here.

The dimmings following the eruption are not well defined and do not allow a reliable determination of their flux content.

\section{Sigmoid reformation}

One of the dominant loops in the northern area of the destroyed sigmoid begins to develop a half $\mathrm{S}$ (or J) shape very soon after the flare arcade has faded; see the image at 11:25 UT in Fig. 10. The following hour leading up to the XRT data gap between 12:28-16:02 UT sees a gradual approach of the southern end of this structure with the J shaped nearest loop in the southern part of the post-event arcade. The images after the data gap show that the two loops merge into a new, continuous $\mathrm{S}$ shaped sigmoid in the same location as the pre-eruption sigmoid, albeit smaller (Fig. 10, panels 3 and 4). The evolution continues to be dynamic with the new sigmoid showing reduced brightness in the middle and a bright spot near this intensity dip for part of the time, as well as a changing intensity ratio between the northern and southern arms and changing sharpness, with a multi-thread appearance at times.

The reformation of the sigmoid and its subsequent dynamics are driven by the changes in the photospheric flux distribution. In particular, the merging into a new continuous $S$ shaped sigmoid is cospatial with the ongoing flux cancellation episode in the range $y \approx-65^{\prime \prime}$ to $-85^{\prime \prime}$ (see the final MDI frame in Fig. 5). Although the amount of cancelled flux in the approximately $6 \mathrm{~h}$ of sigmoid reformation is far smaller than the flux cancelled in
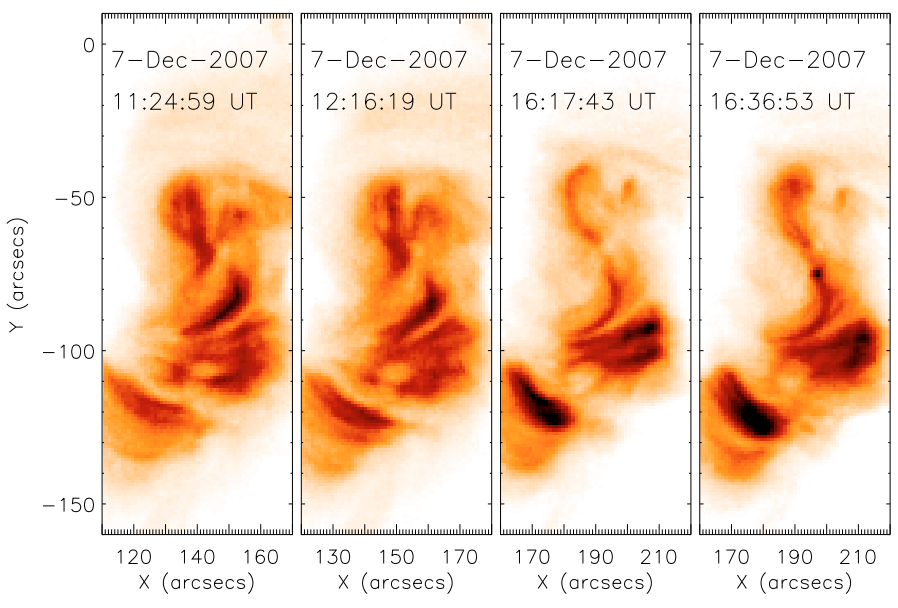

Fig. 10. Hinode/XRT C Poly filter observations showing the reformation of the sigmoid after the successful eruption which produced a CME.

the 2.5 days prior to the eruption (Figs. 1 and 2), a complete new sigmoid is formed. We will discuss this difference in Sect. 6.2.1.

The new sigmoid was destroyed in a very weak eruption, including a CME, on 8 December after 17 UT.

\section{Discussion}

\subsection{Flux rope topology}

The observations of AR 10977 strongly support the picture of the gradual transformation of arcade field into a flux rope as a result of photospheric flux cancellation over extended periods prior to an eruption, as suggested by van Ballegooijen \& Martens (1989). A major flux cancellation episode occurs in the period of 2.5 days between the time of peak flux content after the region's emergence and a CME/flare event. It comprises many cancellation events of flux fragments at the internal PIL. This transforms the initially weakly sheared arcade of soft X-ray loops into a sigmoidal region exhibiting a key signature of a flux rope formed by cancellation: the inverse crossing of the PIL by the middle of the sigmoid, bracketed by two regular PIL crossings by the sigmoid elbows.

The specific magnetic structure of a flux rope can be split into two categories; flux ropes having their underside rooted in the dense lower atmosphere having a bald patch separatric surface (BPSS) topology (Titov \& Démoulin 1999), and flux ropes which are situated in the corona having a so-called hyperbolic flux tube (HFT), i.e., a magnetic X-type structure, at their underside (Titov et al. 2002).

If the reconnection that enables flux cancellation proceeds in or very near the photospheric level, as suggested by van Ballegooijen \& Martens (1989), then it is likely that the flux rope initially forms with BPSS topology. However, in the course of a cancellation episode, the topology can change into one containing an HFT (Aulanier et al. 2010). The transition begins by splitting the bald patch into two sections, with the HFT connecting their inner end points through the corona. The two bald patch sections shrink while the HFT grows in the course of the transition.

BPSS and HFT flux ropes exhibit a completely different behaviour in the event of an eruption. A BPSS flux rope must split in two along a horizontal line, with only the upper part erupting, since its lower part is tied to the lower solar atmosphere 
(Gibson \& Fan 2006). Since sigmoids form in the lower surface of their host flux ropes (Titov \& Démoulin 1999; Green et al. 2007), a sigmoid in a BPSS flux rope forms in the bottom part of the BPSS and is likely to survive the eruption, at least partly. These sigmoids are located below the developing flare current sheet, hence also below the post-eruption arcade. Sigmoids forming in the quasi-separatrix layer in the underside of an HFT flux rope are located in and above the HFT. In an eruption, the HFT pinches into the flare current sheet (Török et al. 2004), so that essentially the whole flux rope above it is ejected and the sigmoid ceases to exist after the eruption.

From the destruction of the sigmoid in the eruption on 7 December 2007 we can thus conclude that the flux rope did likely possess an HFT structure at this time.

\subsection{Magnetic flux budget}

The unsigned flux content of the active region at the time of the eruption is $\approx 1.17 \times 10^{21} \mathrm{Mx}$. Up to this point, a total flux of $0.71 \times 10^{21} \mathrm{Mx}$ is cancelled at the internal PIL, equal to $\approx 34 \%$ of the peak negative flux in the active region prior to the cancellation episode. If all flux lost at the internal PIL caused other flux of the active region to transform into the flux rope in the manner anticipated by van Ballegooijen \& Martens (1989), then $\approx 60 \%$ of the active region flux is in the rope at the time of the eruption. The ratio would be even higher if the flux crossing the PIL south of the sigmoid were excluded from the unsigned flux content of the active region. A flux ratio of this magnitude can still permit force-free equilibrium in model fields (see Fig. 1 in Valori et al. 2010, for examples). However, it differs strongly from the threshold value for the occurrence of force imbalance found in recent numerical investigations of flux rope structure and stability in decaying active regions.

By inserting a flux rope above and along the PIL in the extrapolated potential field of active regions that have undergone significant flux cancellation, and through subsequent numerical relaxation of the configuration, Bobra et al. (2008), Su et al. (2009) and Savcheva \& van Ballegooijen (2009) found a limit for the axial flux in a rope that can be held in stable force-free equilibrium by the other flux of the active region. The limit amounts to at most $10 \%$ of the unsigned flux in the active region for the three regions studied in Bobra et al. (2008) and Su et al. (2009). The limiting axial flux given in Savcheva \& van Ballegooijen (2009) for a fourth, strongly decayed and sigmoidal active region combines with the amount of unsigned flux in the region (which we find to be $3.5 \times 10^{21} \mathrm{Mx}$ ) to a limiting ratio of $\approx 14 \%$.

These three investigations also indicate that the poloidal flux in the rope is not well constrained by their method of comparing field lines in an array of relaxed flux rope configurations with varying axial and poloidal fluxes to observed coronal features. Typically, the poloidal flux could be raised from the best-fit value in a wide range of about an order of magnitude, whilst still fitting the observed structures, until the numerical relaxation failed. Expressing the average twist angle in the flux rope as $\Theta=\pi \Phi_{\text {pol }} / \Phi_{\text {axi }}$, the rope is expected to be stable against the helical kink mode for a poloidal flux of (typically) up to $\Phi_{\mathrm{pol}} \sim 3 \Phi_{\mathrm{axi}}$ (Török et al. 2004; Fan \& Gibson 2004). Indeed, Bobra et al. (2008) find a stable configuration with $\Phi_{\text {pol }} / \Phi_{\text {axi }}=2.6$ (their model 7 for AR 9997/10000) and Savcheva \& van Ballegooijen (2009) even find a stable configuration with $\Phi_{\mathrm{pol}} / \Phi_{\mathrm{axi}}=4.8$ (their model for a sigmoid on 12 Feb. 2007, 08:38 UT). These values indicate that only $\sim 1 / 4$ of the flux in the rope may be axially directed, potentially reducing the discrepancy with our result to a large extent. However, the flux ropes that best fitted the observed active region structure in these investigations were systematically found to have considerably lower amounts of poloidal flux, $\Phi_{\text {pol }} / \Phi_{\text {axi }} \ll 1$ in Bobra et al. (2008) and Su et al. (2009) and 1/4 in Savcheva \& van Ballegooijen (2009), so that other explanations for the discrepancy should be considered.

Observations of interplanetary CMEs which exhibit a flux rope structure indicate a dominance of the poloidal flux over the axial flux, typically by a factor $\sim 3$ (e.g., Mandrini et al. 2005; Attrill et al. 2006; Qiu et al. 2007). However, much of this flux is added to the rope by reconnection after the launch of the CME (Qiu et al. 2007). Therefore, these observations do not provide conclusive evidence of the partition between axial and poloidal flux in the rope at the onset of the eruption.

We note that Sterling et al. (2010) recently also found a considerable amount of cancellation prior to an eruption in another active region. About $20 \%$ of the unsigned active region flux cancelled over two days preceding the event, and further cancellation may have occurred at earlier times not analysed in that study.

In the following, we discuss two aspects of the process of flux rope formation by cancellation which may help reconcile the discrepancy between our measurements of the amount of flux at the onset of eruption in Sect. 2 and the results of the numerical modelling based on the flux rope insertion method.

\subsubsection{Cancelled flux versus rope flux}

van Ballegooijen \& Martens (1989) argued that magnetic flux can submerge below the photosphere only if it forms small loops with a radius of curvature comparable to the photospheric pressure scale height, giving an estimated maximum distance between the loop footpoints of $\sim 900 \mathrm{~km}$. If the flux in the active region has shear, most loops in the original arcade will have a footpoint separation in the direction of the PIL larger than this value. This is definitely the case for AR 10977 from the onset of the cancellation episode (see Fig. 3, panel 1). When the footpoints of these loops are transported toward the PIL, the loops remain too long for submergence. Rather they reconnect with other loops at, or somewhat above, the photospheric PIL, creating the short loops which can submerge and long loops rooted away from the cancellation site at the remote footpoints of the reconnecting loops. The downward tension force of the long loops is insufficient to drive them below the photosphere, and their middle part tends to be more aligned with the PIL than the original two loops. Therefore, cancellation leads to an accumulation of flux which runs along the active section of the PIL, where flows converging toward the PIL drive the cancellation episode and where sufficient flux exists on both sides to enable cancellation events.

If axial flux already exists above the PIL (which can result from previous cancellation) then the newly reconnected long loops are helical. They wrap around the bottom side of the existing axial flux such that a flux rope gradually forms as the cancellation episode continues.

If only a single cancellation event occurs, then, by the nature of the process, the flux accumulated at the PIL equals the cancelled flux. However, this is no longer the case when subsequent cancellation occurs which involves the product of a previous event. In this case, no further flux is added, rather the accumulated flux will extend further along the PIL. An amount of flux equal to the flux in the long loop is lost from the system. This is illustrated by the schematic in Fig. 11. The effect can also be seen in the filament formation scenario proposed in 

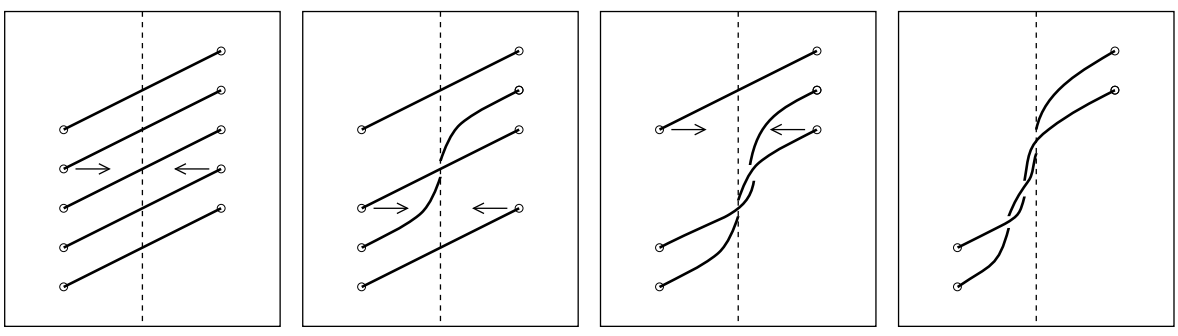

Fig. 11. Schematic of a cancellation episode which consists of three cancellation events and transforms the part of the initial arcade shown in the first panel into a flux rope. The PIL is drawn dashed. The ratio of footpoint displacement of the sheared arcade loops to the length of the active section of the PIL (where cancellation proceeds) is $2: 3$. Correspondingly, $2 / 5$ of the flux is transformed into the rope, while $3 / 5$ cancel.
Martens \& Zwaan (2001, their Fig. 6), but has not been explicitly addressed in that paper.

Thus, of the original loops that pass over the active section of the PIL, three categories must be distinguished: loops rooted at one end in flux that cancels, loops rooted at both ends in flux that cancels, and loops rooted in non-cancelling flux at sufficient distance to the PIL. Half of the flux in the first category will be transformed into the flux that accumulates at the PIL and eventually forms a flux rope, while the other half cancels. The flux in the second category will be lost without contributing to the amount of flux in the rope. The third category remains in the active region as overlying, stabilizing flux.

As can be seen from Fig. 11, the ratio between the flux accumulated at the PIL and the cancelled flux depends on the shear of the original arcade and on the length of the active section of the PIL. For roughly uniform flux along the active section of the PIL, it is given by the ratio between the displacement of the loop footpoints parallel to the PIL and the length of the active section (with an upper limit of unity), remaining small for a weakly sheared arcade.

This ratio can change considerably in the course of a cancellation episode, by changing shear as well as by changing length of the active PIL section. Typically, the shear increases and the length of the active PIL section decreases when large parts of the flux in an active region cancel. Thus, the process of flux transformation from arcade to flux rope due to cancellation accelerates. Therefore, it seems plausible that sigmoids are typically observed late in a cancellation episode, closer to an eruption than to the onset of the cancellation, as in the event studied here, and that the occurrence of sigmoids has such a high correlation with eruptive activity (Canfield et al. 1999).

Figure 2 shows that the shear increases while the flux in AR 10977 cancels. The change in the length of the active PIL section is less pronounced. The shear remains weak during 4 December, but grows considerably on 5 December, which is also clearly visible in the soft X-ray images in Fig. 3. Still, the shear remains moderate until about mid-day on 5 December, by which time $\approx 40 \%$ of the cancellation has occurred (Table 1 ). Most of this flux and part of the subsequently cancelled flux is lost from the active region without contributing to the amount of flux in the rope. This reduces the discrepancy to the numerically obtained stability limit by a factor $\sim 2$, or somewhat higher.

The sigmoid reformation after the CME started in a region of highly sheared flux, as evidenced by the northern set of posteruption loops (images at 10:23-11:25 UT in Figs. 8 and 10). Therefore, a new flux rope and sigmoid could be formed through an amount of cancellation much less than the cancellation in the period prior to the eruptions on 7 December.

\subsubsection{Coherence of the flux rope}

A further cause for the discrepancy may be found in the differing degree of coherence in the flux rope structure. The flux rope inserted in the three numerical studies mentioned above is of course fully coherent along its length and unlikely to lose much of this coherence in the relaxation. (Since a vacuum sheath surrounding the flux rope was also inserted, both the external incoherent field and the inserted flux rope could expand in the relaxation to form a current layer, where much of the structural differences between the flux rope and its exterior could be resolved, so that these did not strongly propagate into the flux rope.) In order to form such a coherent flux rope by cancellation, the cancellation must proceed largely uniformly along a substantial fraction of the length of the resulting rope. Only then will concave-up field lines exist along a substantial fraction of its length, and only then will field lines spiral above the middle part of the rope (these field lines must be concave-up near one or both ends of the rope). The observations, however, indicate that the cancellation episode evolved from an initially more uniform distribution of cancellation events along the active section of the PIL to a more localised pattern (compare panels 1-3 in Fig. 2 with panels 4-5). At the same time, the length of the sigmoid increased. The cancellation was by far strongest in the middle of the forming sigmoid where the inverse PIL crossing occurred. At this position the number of helical field lines running under the axis of the forming flux rope is much larger than the number of helical field lines passing over the axis.

As discussed in Sect. 3, the linear bar-like feature is suggestive to be of arcade structure prior to the eruption, simply passing over the gradually forming flux rope without already being a part of it. It passes over the sigmoid's inverse PIL crossing, where most rope field lines are upward concave. By partly joining the sigmoid, the linear feature adds downward concave flux in this location, leading to a more complete and more coherent flux rope structure. The temporal association of this process with the onset of the successful eruption is likely no coincidence. Also, the coherence of the flux rope is likely completed only in the process of the eruption.

\section{Summary and conclusions}

We present observations of NOAA active region 10977 which was observed from emergence through to the decay phase, during which a coronal mass ejection occurred. The evolution of the magnetic field in the decay phase was dominated by fragmentation of the photospheric field, the development of supergranular flows, and cancellation of photospheric flux fragments at the developing supergranular boundaries. The observations support the interpretation that a major flux cancellation episode was responsible for the formation of a flux rope in the active region during the $\sim 2.5$ days preceding the CME. A soft X-ray sigmoid, crossing the PIL in the inverse direction, suggests that a flux rope has formed prior to the eruption. The sigmoid was destroyed by the eruption, indicating that the flux rope had developed a hyperbolic flux tube (HFT) at its underside. Continued cancellation 
through and after the eruption led to the quick reformation of the sigmoid, which later erupted again.

The unsigned flux in the active region at the time of the eruption is about $1.17 \times 10^{21} \mathrm{Mx}$. During the 2.5 day period before the eruption about $0.71 \times 10^{21} \mathrm{Mx}$ cancels at the internal PIL of the active region, equal to $\approx 34 \%$ of the peak negative flux in the active region prior to the cancellation episode. If the reconnection which enables the flux cancellation transformed an equal amount of arcade flux into the flux of the forming rope according to the mechanism put forward by van Ballegooijen \& Martens (1989), then the rope would contain about $60 \%$ of the active region flux. This is much larger, by a factor $\approx 4-6$, than the limiting axial flux value which would maintain force balance in a decaying active region, as found in recent numerical modelling (Bobra et al. 2008; Su et al. 2009; Savcheva \& van Ballegooijen 2009; Aulanier et al. 2010; Amari et al. 2010).

We point out that the amount of flux transformed from the initial arcade into the flux rope can differ considerably from the amount of cancelled flux. If the initial arcade is only weakly sheared, then the amount of arcade flux rooted beyond the ends of the active section of the PIL is far smaller than the amount of flux at both sides of the active section. The former is the flux available for transformation into the flux rope. The latter can cancel without contributing to the flux in the rope. Their ratio is (very roughly) given by the ratio between the displacement of the arcade loop footpoints parallel to the PIL and the length of the active section of the PIL, limited to unity. Thus, for an initially only weakly sheared arcade, the amount of flux cancelled can be much larger than the amount of flux transformed into the flux rope.

If the cancellation occurs predominantly along a section of the PIL which is small in relation to the extent of the forming flux rope, as in the late phase of the studied event, then most of the helical, concave-up field lines have their bottom section in this localized area. Helical field lines passing over this area are strongly underrepresented, so that the resulting flux rope is incoherent to a considerable degree. This is likely to contribute to the discrepancy with the numerically obtained stability limit. Also, it suggests that flux ropes built by flux cancellation often reach coherence only in the course of an eruption.

The sigmoid forming in AR 10977 includes a linear, barlike feature, which is hotter than the $S$ shaped threads prior to the eruption. In this phase, the feature must be interpreted as a bundle of arcade field lines passing over the developing flux rope. The data yield evidence that this flux becomes part of the flux rope immediately before and in the early stages of the eruption, through the cancellation at its southern end and the further reconnection which establishes the coherence of the flux rope. Subsequently it traces the path of the erupting flux rope body, as suggested for similar features in earlier events (Moore et al. 2001; McKenzie \& Canfield 2008).

Acknowledgements. We thank the referee, G. Aulanier, for the very constructive reports that significantly helped improve the clarity of this paper and Duncan Mackay for helpful discussions and comments on the manuscript. L.M.G. was supported by a Royal Society Dorothy Hodgkin Fellowship and a Leverhulme Fellowship. A.J.W. was supported by STFC via a Ph.D. studentship. B.K. acknowledges support by the DFG, the STFC, and NASA grants NNH06AD58I and NNX08AG44G. Hinode is a Japanese mission developed and launched by ISAS/JAXA, collaborating with NAOJ as a domestic partner, NASA and STFC (UK) as international partners. Scientific operation of the Hinode mission is conducted by the Hinode science team organised at ISAS/JAXA. This team mainly consists of scientists from institutes in the partner countries. Support for the post-launch operation is provided by JAXA and NAOJ (Japan), STFC (UK), NASA (USA), ESA, and NSC (Norway). This research has made use of the LASCO CME catalog, generated and maintained at the CDAW Data Center by NASA and The Catholic University of America in cooperation with the NRL. SOHO is a project of international cooperation between ESA and NASA.

\section{References}

Amari, T., Luciani, J. F., Aly, J. J., Mikic, Z., \& Linker, J. 2003, ApJ, 595, 1231 Amari, T., Aly, J., Mikic, Z., \& Linker, J. 2010, ApJ, 717, L26

Antiochos, S. K., Dahlburg, R. B., \& Klimchuk, J. A. 1994, ApJ, 420, L41

Athay, R. G., Querfeld, C. W., Smartt, R. N., Landi Degl'Innocenti, E., \& Bommier, V. 1983, Sol. Phys., 89, 3

Attrill, G., Nakwacki, M. S., Harra, L. K., et al. 2006, Sol. Phys., 238, 117

Aulanier, G., Török, T., Démoulin, P., \& DeLuca, E. E. 2010, ApJ, 708, 314

Bellot Rubio, L. R., \& Beck, C. 2005, ApJ, 626, L125

Bobra, M. G., van Ballegooijen, A. A., \& DeLuca, E. E. 2008, ApJ, 672, 1209

Brown, C. M., Feldman, U., Seely, J. F., Korendyke, C. M., \& Hara, H. 2008, ApJS, 176, 511

Canfield, R. C., Hudson, H. S., \& McKenzie, D. E. 1999, Geophys. Res. Lett., 26,627

Canou, A., Amari, T., Bommier, V., et al. 2009, ApJ, 693, L27

Chen, F., \& Ding, M. D. 2010, ApJ, 724, 640

Culhane, J. L., Harra, L. K., James, A. M., et al. 2007, Sol. Phys., 243, 19

Fan, Y. 2001, ApJ, 554, L111

Fan, Y., \& Gibson, S. E. 2004, ApJ, 609, 1123

Forbes, T. G., \& Isenberg, P. A. 1991, ApJ, 373, 294

Gibson, S. E., \& Fan, Y. 2006, ApJ, 637, L65

Golub, L., Deluca, E., Austin, G., et al. 2007, Sol. Phys., 243, 63

Green, L. M., \& Kliem, B. 2009, ApJ, 700, L83

Green, L. M., Démoulin, P., Mandrini, C. H., \& Van Driel-Gesztelyi, L. 2003, Sol. Phys., 215, 307

Green, L. M., Kliem, B., Török, T., van Driel-Gesztelyi, L., \& Attrill, G. D. R 2007, Sol. Phys., 246, 365

Harvey, K. L., Jones, H. P., Schrijver, C. J., \& Penn, M. J. 1999, Sol. Phys., 190, 35

Hood, A. W., \& Priest, E. R. 1981, Geophys. Astrophys. Fluid Dyn., 17, 297

Jian, L., Russell, C. T., Luhmann, J. G., \& Skoug, R. M. 2006, Sol. Phys., 239, 393

Kliem, B., \& Török, T. 2006, Phys. Rev. Lett., 96, 255002

Lites, B. W. 2005, ApJ, 622, 1275

Ma, S., Wills-Davey, M. J., Lin, J., et al. 2009, ApJ, 707, 503

Mackay, D. H., \& van Ballegooijen, A. A. 2006, ApJ, 641, 577

Mandrini, C. H., Pohjolainen, S., Dasso, S., et al. 2005, A\&A, 434, 725

Martens, P. C., \& Zwaan, C. 2001, ApJ, 558, 872

Martin, S. F., Livi, S. H. B., \& Wang, J. 1985, Aust. J. Phys., 38, 929

McKenzie, D. E., \& Canfield, R. C. 2008, A\&A, 481, L65

Moore, R. L., Sterling, A. C., Hudson, H. S., \& Lemen, J. R. 2001, ApJ, 552, 833

Pevtsov, A. A., Canfield, R. C., \& McClymont, A. N. 1997, ApJ, 481, 973

Qiu, J., Hu, Q., Howard, T. A., \& Yurchyshyn, V. B. 2007, ApJ, 659, 758

Savcheva, A., \& van Ballegooijen, A. 2009, ApJ, 703, 1766

Scherrer, P. H., Bogart, R. S., Bush, R. I., et al. 1995, Sol. Phys., 162, 129

Sterling, A. C., Chifor, C., Mason, H. E., Moore, R. L., \& Young, P. R. 2010 A\&A, 521, A49

Su, Y., van Ballegooijen, A., Lites, B. W., et al. 2009, ApJ, 691, 105

Titov, V. S., \& Démoulin, P. 1999, A\&A, 351, 707

Titov, V. S., Hornig, G., \& Démoulin, P. 2002, J. Geophys. Res., 107, 1164

Titov, V. S., Priest, E. R., \& Demoulin, P. 1993, A\&A, 276, 564

Török, T., \& Kliem, B. 2005, ApJ, 630, L97

Török, T., Kliem, B., \& Titov, V. S. 2004, A\&A, 413, L27

Tripathi, D., Kliem, B., Mason, H. E., Young, P. R., \& Green, L. M. 2009, ApJ, 698, L27

Valori, G., Kliem, B., Török, T., \& Titov, V. S. 2010, A\&A, 519, A44

van Ballegooijen, A. A., \& Martens, P. C. H. 1989, ApJ, 343, 971

van Tend, W., \& Kuperus, M. 1978, Sol. Phys., 59, 115

Wang, Y., \& Muglach, K. 2007, ApJ, 666, 1284

Webb, D. F., Lepping, R. P., Burlaga, L. F., et al. 2000, J. Geophys. Res., 105, 27251

Yurchyshyn, V. B., \& Wang, H. 2001, Sol. Phys., 202, 309

Zwaan, C. 1987, ARA\&A, 25, 83 